\title{
Atomic Scale Dynamics of a Manganese Oxide Phase Change Observed with STEM
}

\author{
Timothy J. Pennycook ${ }^{1,2}$, Lewys Jones ${ }^{1}$, Henrik Pettersson ${ }^{3}$, Valeria Nicolosi ${ }^{3}$ and Peter D. Nellist ${ }^{1,2}$ \\ 1. Department of Materials, University of Oxford, Parks Road, Oxford OX1 3PH, UK \\ 2. SuperSTEM Laboratory, Daresbury, WA4 4AD, UK \\ 3. Trinty College Dublin, College Green, Dublin 2, IR.
}

When studying a dynamic system, time resolution can be as important as spatial resolution. In scanning transmission electron microscopy (STEM) the time resolution is predominately dependent on the dwell time and the number of pixels used to sample a given region. Consider a dynamic process involving a multitude of successive steps, each of which requires overcoming an energy barrier. The necessary energy for this process could come from heat, or it could come from an electron probe. In the latter case, the rate this process unfolds will depend on the energies of the beam and barrier and the amount of time the probe is very near where the process is occurring. In aberration corrected STEM it is possible to record atomic resolution images with each pixel sampled on the order of a microsecond and relatively few pixels per atomic column. Thus for dynamic processes with appropriate energy barriers STEM can be used as a pump-probe experiment. The atomic number contrast of high angle angular dark field (HAADF) STEM allows one to differentiate atomic species and makes possible the interpretation of dynamic changes in atomic column intensities and shape. Furthermore, by recording a time series of images it is possible to correct for sample drift. Drift corrected frames can also be summed, avoiding motion blur associated with long dwell times. Time resolved HAADF imaging has previously uncovered differences in the dynamics of small and ultrasmall semiconductor nanoclusters that explained white light emission from ultrasmall sub-2-nm CdSe nanoclusters [1]. In the present work we demonstrate the use of time resolved HAADF imaging to unveil the detailed atomic motions involved in a phase change.

Fig $1 \mathrm{~A}$ shows a HAADF image of the edge of a very thin flake of spinel $\mathrm{Mn}_{3} \mathrm{O}_{4}$. By scanning the electron probe over a region at the edge of the flake for an extended period of time we provided the energy needed to convert the $\mathrm{Mn}_{3} \mathrm{O}_{4}$ in this region into another manganese oxide phase as shown in Fig. 1B. The structure of the two phases and their interface is shown in more detail in Fig. 2, which displays two images each formed from the average of nine successive frames from a drift corrected 512 by 512 pixel HAADF movie with a dwell time of 2.6 microseconds per pixel and a flyback time of 150 microseconds, equating to $\sim 0.75 \mathrm{~s}$ per frame. Fifty seven frames were recorded between those forming Fig. $2 \mathrm{~A}$ and B, so the two images are separated in time by $\sim 43 \mathrm{~s}$. During this time the phase front has moved down just three monolayers, meaning we have about 19 frames per monolayer advance of the phase front.

$\mathrm{Mn}_{3} \mathrm{O}_{4}$ is a mixed valance material, containing both $\mathrm{Mn}^{3+}$ and $\mathrm{Mn}^{2+}$ in the formation illustrated in Fig. 2A [2]. The bright $\mathrm{Mn}^{3+}$ columns contain double the number of $\mathrm{Mn}$ atoms as the darker $\mathrm{Mn}^{3+} \mathrm{and} \mathrm{Mn}^{2+}$ columns. The phase change consists of the reconstruction of the 10 darker columns surrounding each bright Mn column into 8 bright Mn columns. A series of images each formed by averaging four frames from the same movie used to create Fig. 2 illustrate this process in Fig. 3. The first row of dark atoms below the consistently bright atoms of the new phase in Fig. 3A is indicated with a pair of white arrows. A red arrow points to a single member of this row of $\mathrm{Mn}^{3+}$ columns, and a green arrow to a pair of $\mathrm{Mn}^{2+}$ columns in the next row down. These arrows are placed on the same position in all further panels of Fig. 
3 to provide a reference for the eye. During the second of the four frames composing Fig. 3B, part of the intensity of the $\mathrm{Mn}^{2+}$ column next to the blue arrow displaces up towards the $\mathrm{Mn}^{3+}$ (shown in the inset) after which the $\mathrm{Mn}^{2+}$ column has lost intensity and the $\mathrm{Mn}^{3+}$ column gained intensity, suggesting that the $\mathrm{Mn}^{2+}$ column has donated atoms to it. Next the pair of $\mathrm{Mn}^{2+}$ columns nearest the blue arrow move towards each other, oscillating around the midpoint between them. In Fig. 3 these oscillations appear as lateral blurs partly due to each image here being the average of 4 movie frames. As they oscillate, further displacements of the $\mathrm{Mn}^{2+}$ columns towards the $\mathrm{Mn}^{3+}$ columns in the row above occur, and the intensity of the $\mathrm{Mn}^{3+}$ columns increases, suggesting the oscillations are transporting $\mathrm{Mn}$ atoms up into in the $\mathrm{Mn}^{3+}$ columns at the phase front. When these $\mathrm{Mn}^{3+}$ columns reach the intensity of the bright spinel columns, the motion of the $\mathrm{Mn}^{2+}$ pair stops and they coalesce into a single bright column. These motions are more apparent in the full movie and are seen repeatedly across the phase front as it expands. We will present these movies and more detailed quantitative analysis of the dynamics of the positions and intensities of the columns during the phase change. In addition we will show how electron energy loss spectroscopy analysis can provide further insight into the process. The fine structure of the O K- and Mn L-edges convincingly identify the new phase as the rock salt structure of $\mathrm{MnO}$ [3].

\section{References:}

[1] T. J. Pennycook et al, Nanoletters 12 (2012) p. 3038.

[2] A. Tan et al, Phys. Rev. Lett. 107 (2011) p. 107602.

[3] This research was supported by the EPSRC through the UK National Facility for AberrationCorrected STEM (SuperSTEM) and the ERC starting grant 2DNanoCaps (VN and HP).

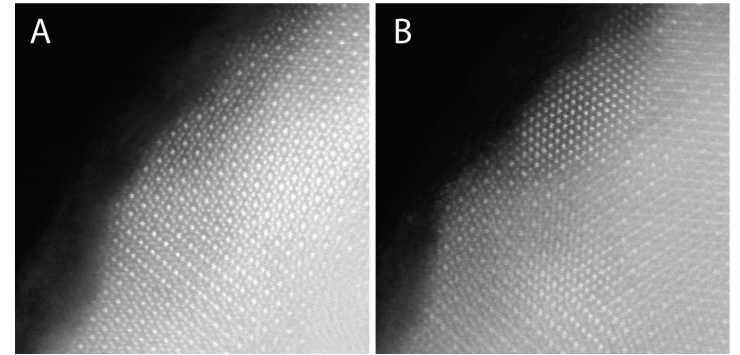

Figure 1. HAADF images before and after nucleation of the new phase at the edge of spinel $\mathrm{Mn}_{3} \mathrm{O}_{4}$.

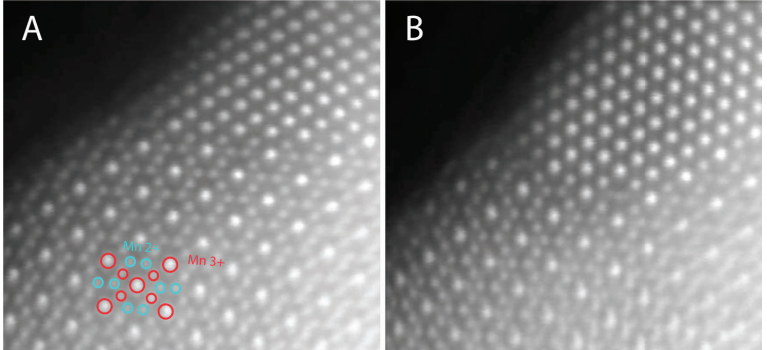

Figure 2. HAADF images showing the expansion of the new phase into the spinel. The overlay on A illustrates which columns are $2+$ and which are $3+$ in the spinel.
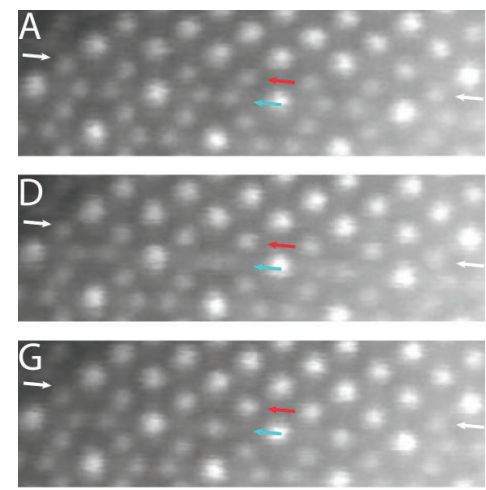
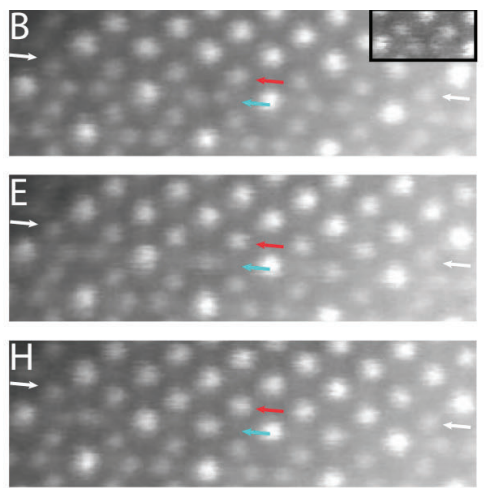
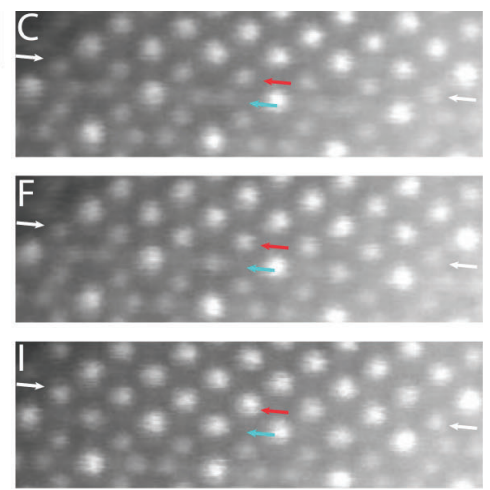

Figure

3. Sequential series of images each created from four frames of a HAADF movie of the phase change. The white arrows indicate the to the position in A of a single column of $\mathrm{Mn}^{3+}$ and pair of $\mathrm{Mn}^{2+}$ columns respectively. 